\title{
74. NEUTRAL HYDROGEN IN THE SAGITTARIUS AND SCUTUM SPIRAL ARMS
}

\author{
W. B. BURTON and W. W. SHANE \\ Leyden University Observatory, Leyden, The Netherlands
}

\begin{abstract}
Observations of the neutral hydrogen in the first quadrant of galactic longitude have been analysed. The existence of large-scale streaming motions such as the streaming associated with the Sagittarius arm makes interpretation of the observations in terms of circular galactic rotation unsatisfactory. It is shown that application of the density-wave theory formulated by Lin et al. (1969) leads to a more satisfactory interpretation. Using kinematic models based on this theory the distribution and motion of the neutral hydrogen are studied. Failures of kinematic models based on circular rotation are pointed out. A map of the distribution of neutral hydrogen is produced. The Scutum arm is composed of inner and outer arcs both of which seem to be moving outward from the galactic center with velocities of the order of $30 \mathrm{~km} \mathrm{~s}^{-1}$.
\end{abstract}

\section{Analysis of the Observations}

Profiles of the 21-cm line of $\mathrm{HI}$ have been obtained using the 25-m telescope in Dwingeloo, covering the range of (new) galactic longitude $l^{\mathrm{H}}=22^{\circ}$ to $56^{\circ}$ and extending about five degrees on either side of the galactic plane. The observations are generally complete for positive velocities and were made with a bandwidth of 2 or $4 \mathrm{~km} \mathrm{~s}^{-1}$. These observations, which constitute the principal material for the investigations described here, were analysed in detail. We have also made use of additional observations by S.C. Simonson covering some regions closer to the direction of the galactic center and have drawn on a number of other programs in order to complete the survey of the galactic plane from $l^{\mathrm{II}}=-3^{\circ}$ to $70^{\circ}$. The observations have been represented as contour maps showing isophotes of brightness temperature in the velocity-longitude and velocity-latitude planes.

We have found it convenient in analysing the line profiles to decompose them into Gaussian components. Before doing so, however, we applied two significant corrections. Firstly, we reduced the measured brightness temperatures to optical depths assuming a spin temperature $T_{s}=135 \mathrm{~K}$. We did this reluctantly, realizing that the conditions under which such a correction is valid are by no means fulfilled. However the alternative, making no correction before decomposition into additive components, is equivalent to assuming an infinite spin temperature. We argue that the modest correction implied by our choice of a rather high spin temperature is probably an improvement over no correction at all. Secondly, we have, in those regions where it appeared to be of significance, subtracted a contribution which arises from a rather uniform layer of $\mathrm{HI}$ with a concentration to the galactic plane somewhat less than that of the major spiral features. This feature, which we call the galactic envelope, will not be discussed in this contribution. Its presence in the uncorrected line profiles led to difficulties in the analysis.

The method of Gaussian analysis was chosen primarily as a matter of convenience. 
We were able in this way to describe quantitatively the significant features in the line profiles and to reduce the amount of data by about a half order of magnitude without substantial loss of information.

In most parts of the observed region the Gaussian analysis was performed twice, first for each profile separately in order to identify the structural features and then a second time, introducing the identified features into all profiles at a single longitude, in order to study their dependence upon latitude. At some of the higher longitudes where the line profiles show the least complexity, this double analysis was not required.

It was now possible to determine the dependence of optical depth, velocity, and dispersion in velocity, upon latitude. The first of these could be represented by one or more Gauss functions and the others by polynomials. After a slight further simplification it was possible to describe all of the observations at a single longitude by a sum of bivariate normal functions. Comparison of this distribution with the corresponding contour map of brightness temperature as a function of latitude and velocity shows that all of the features visible on the latter are represented quantitatively by the former.

The large number of components required to give an acceptable representation of the data, as many as twenty at one longitude, reflects the complexity of the detailed structure. Even more detail is visible on high resolution surveys. In order to derive a picture of the most general features of spiral structure it was necessary to combine those components which appeared to be related to a single feature of the order of size of a spiral arm. In order to do this we examined in detail the behavior of each component as a function of longitude as well as the latitude distribution, which also provided the principal criterion for resolution of the distance ambiguity. The high resolution Maryland-Green Bank 21-cm Line Survey was indispensable in following the structure through the complex region near the plane at lower longitudes. In this step, as elsewhere, frequent reference was made to the observed line profiles in order to avoid being misled through the complexity of the analysis. In the higher longitude region, where the profiles were less complex, this part of the analysis presented little difficulty.

Each feature studied in this way can be described in detail, but description through the parameters derived from the above analysis is more useful. These parameters represent maximum optical depth, mean position in latitude and in velocity, dispersion in latitude and in velocity, and rate of change of mean velocity with latitude. Of these, the mean velocity is the most significant, and is plotted, for all the major features identified, in the velocity-longitude diagram (Figure 1). The shaded bands represent the observed features, as described in the caption. The figure is seen to be dominated by two major loops, which we suppose to represent the Sagittarius and Scutum spiral arms. But before we can discuss the space distribution of the neutral hydrogen, we must derive a suitable model on the basis of which we can determine kinematic distances.

\section{Interpretation of the Velocity-Longitude Diagram}

Interpretation of the velocity-longitude diagram requires answers to two questions, which cannot be considered separately: 
(i) what are the characteristics of the velocity field of the Galaxy; and (ii) how is the hydrogen distributed in space throughout the Galaxy.

As a first step we assume that the hydrogen rotates in circular orbits according to a velocity field described by a smooth rotation curve, $\Theta_{c}(R)=250.0+4.05(10-R)-$ $1.62(10-R)^{2}$, where $R$ is distance from the galactic center expressed in kpc. This

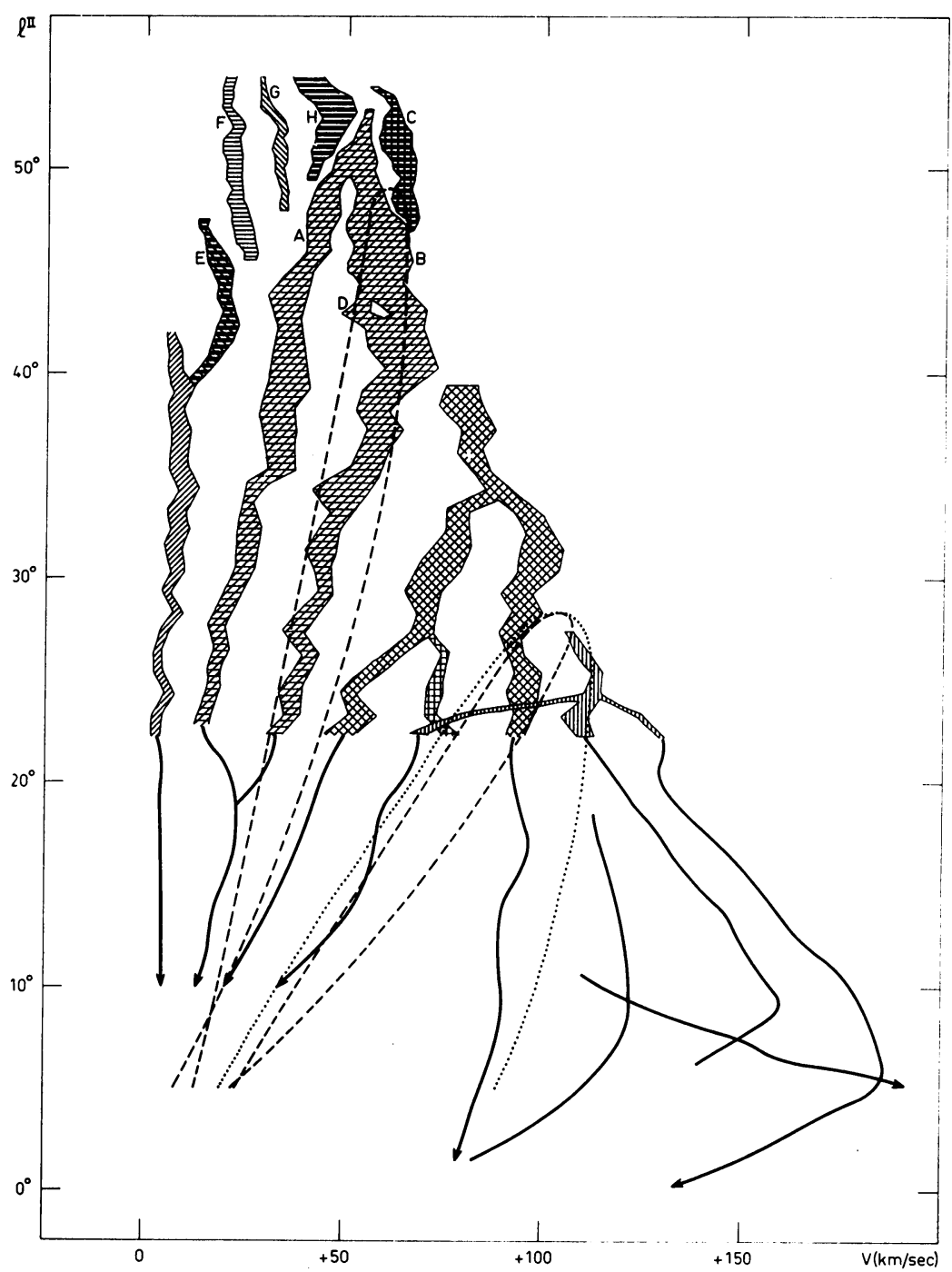

Fig. 1. Observed velocity-longitude diagram for $22^{\circ} \leqslant l \mathrm{II} \leqslant 56^{\circ}$. Separate features are identified by different sorts of shading and, in the upper range of longitude, by letters. The breadth of a feature in velocity is proportional to the integrated $\mathrm{HI}$ content along a line of sight at its central latitude. The diagram has been extended schematically to lower longitudes on the basis of a preliminary analysis by S. C. Simonson. The broken curves represent the axes (density maxima) of the Sagittarius and Scutum arms based on model I. The dotted curve represents the same for the Scutum arm based on model IV. 
basic rotation curve differs by only a few $\mathrm{km} \mathrm{s}^{-1}$ from that of Kwee et al. (1954). Furthermore we suppose a one-to-one correspondence between the velocity-longitude patterns in Figure 1 and concentrations of hydrogen in space.

First step: $\Theta_{c}(R) \& V\left(l^{\text {II }}\right) \Rightarrow$ Map.

To illustrate some of the problems encountered using these simple assumptions we consider the three features labelled $\mathrm{A}, \mathrm{B}$ and $\mathrm{C}$ in the velocity longitude diagram. Features $\mathrm{A}$ and $\mathrm{B}$ are respectively the near and far branches of the Sagittarius arm, and feature $\mathrm{C}$ is the high-velocity stream of gas located on the outside of the Sagittarius arm (Burton, 1966a). The central velocities of these three features are extracted from Figure 1 and replotted as dots in the left side of Figure 2. If the velocities of the Sagittarius arm patterns A and B are converted to distance using the basic rotation curve then the vicinity of the subcentral-point circle in the resulting map is empty, which is certainly not a realistic situation. On the other hand, the velocities of the streaming feature $\mathrm{C}$ are larger than allowed by the basic rotation curve, so that following the assumptions of the first step there is no solution for distance to this feature.

In order to avoid forbidden velocities and the unrealistic situation of the empty subcentral-point region, we depart from the first procedure and introduce flexibility into both the velocity field and the density distribution.

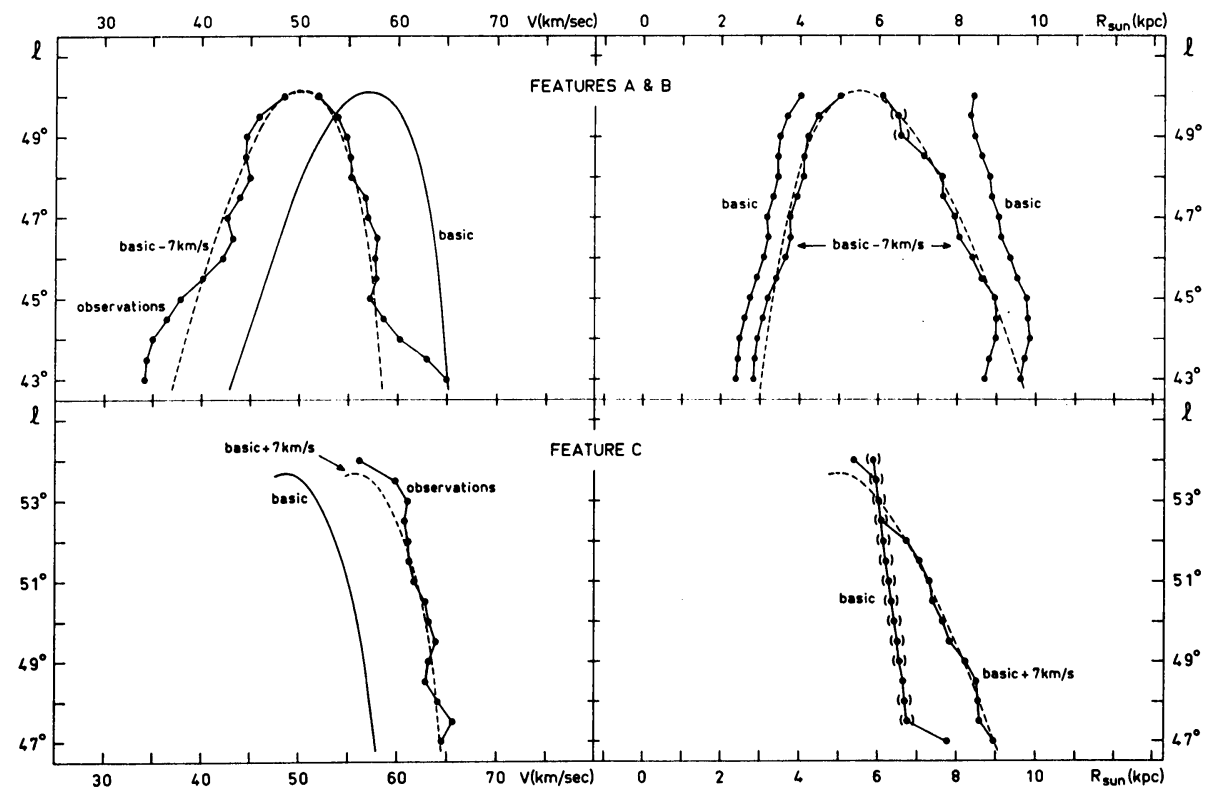

Fig. 2. The observed velocities of features A, B and C, and kinematically derived distances from the Sun. The velocities are taken from Figure 1. Distances are derived by using the basic rotation curve or a shifted rotation curve. Dots in parentheses correspond to forbidden velocities and are plotted at the distance of the subcentral point. The smooth loops show the velocities and distances, based on the indicated rotation curve, for exponential spirals chosen to fit the observations. 
In the second step we abandon circular rotation. We use the same basic rotation curve but postulate for each feature additional motion perpendicular to galactic radii, superimposed on the basic rotation.

Second step: $\Theta_{c}(R)+$ perturbation $\& V\left(l^{\mathrm{II}}\right) \Rightarrow$ Map.

The streaming is adjusted until the space distribution is reasonable. In the case of the Sagittarius arm features A and B, the subcentral-point region is not empty if a streaming of $7 \mathrm{~km} \mathrm{~s}^{-1}$ against galactic rotation is superimposed on the basic rotation. In the case of feature $\mathrm{C}$, by postulating a streaming in the direction of the galactic rotation of $7 \mathrm{~km} \mathrm{~s}^{-1}$ superimposed on the basic rotation, the velocities are not forbidden and the distance of the feature from the Sun can be calculated.

The fifteen year old Kootwijk map was drawn assuming pure circular rotation. In the case of the Kootwijk map it was the density distribution which was adjusted when necessary; the velocity-field assumptions were not relaxed. For example, intensities found at velocities greater than allowed by the assumed rotation curve were smeared along $2 \mathrm{kpc}$ of line of sight, symmetric with respect to the subcentral point. Accordingly, large-scale streaming motions may appear on the resulting map as spurious spiral arms or spiral-like fragments.

A map of the region $43^{\circ} \leqslant l^{I I} \leqslant 56^{\circ}$ drawn following the procedures of the second step is shown in Figure 3. Such a map is necessarily composite since different perturbations to the basic rotation curve will in general be necessary to acccount for the behavior of the various patterns in the velocity-longitude diagram. There are also patterns for which there is no reason to adopt any deviation from the basic rotation. When the distance ambiguity has to be resolved, as in preparing Figures 2, 3 and 12, the distribution in latitude serves as the primary criterion. Where available, absorption measurements are also helpful.

In a third step we will try to interpret the observed streaming motions in terms of the first-order density-wave theory as formulated by Lin et al. (1969).

Third step: $\Theta_{c}(R)+$ density-wave perturbations $\& V\left(l^{\mathrm{II}}\right) \Rightarrow$ Models $\&$ Map.

\section{Model Calculations}

A contour map of hydrogen in the galactic plane is shown in Figure 4. This map was produced in cooperation with S. C. Simonson. The models may be compared with this reference map, although our interpretation is based on analysis of many more measurements. It is evident from the map that the run of terminal velocities with longitude shows irregularities. The bump around $l=52^{\circ}$ is associated with the Sagittarius spiral arm; the other main bump, around $l=32^{\circ}$, is associated with the Scutum arm. A more or less continous spiral arm appears at negative velocities, and an arm outside the Sagittarius arm appears at velocities near zero, superimposed on local gas. Regions of minimum intensity in the contour map can be just as important in the analysis as regions of high intensity. The minimum intensity regions between the 
Sagittarius arm and the Scutum arm, and between the Sagittarius arm and the arm outside it (at velocities near zero), are both evident in the reference map.

Model I is based on the first-order density-wave theory and is represented as a contour map in Figure 5. The axis of the Sagittarius arm is seen tangentially at $l^{\mathrm{II}}=50^{\circ}$ in the model and the axis of the Scutum arm is seen tangentially at $l^{\mathrm{II}}=29^{\circ}$. Non-circular arms located closer to the galactic center than $10 \mathrm{kpc}$ will appear as loops in velocitylongitude space, but resolution of the near and far branches of these arms is generally prevented in the models by the large contour interval.

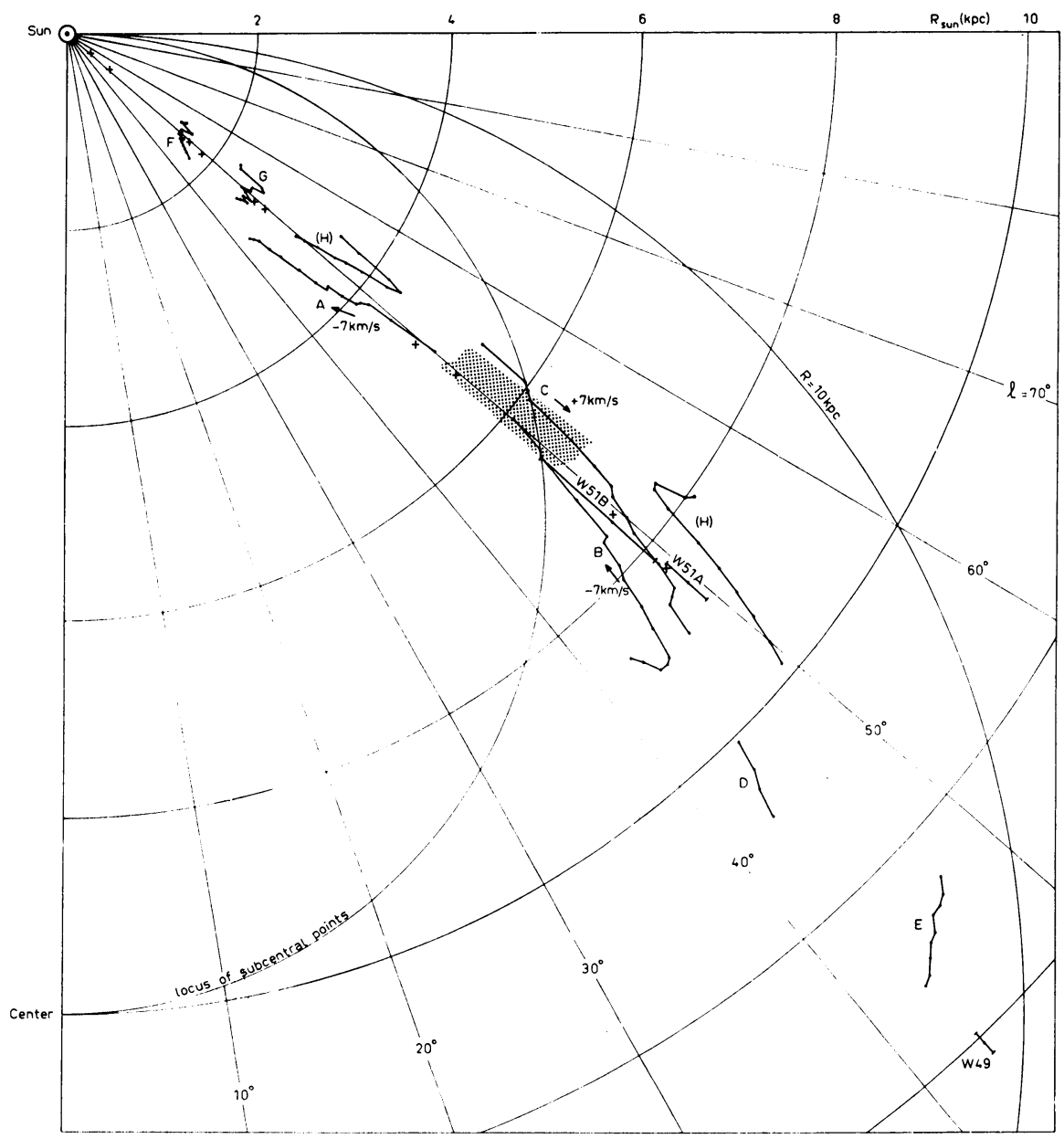

Fig. 3. Map of $\mathrm{HI}$ distribution near the galactic plane in the region $43^{\circ} \leqslant l^{\mathrm{II}} \leqslant 56^{\circ}$. The kinematic distances to the Sagittarius arm features $\mathbf{A}$ and B, and to the high-velocity feature C, are based on the basic rotation curve plus a perturbation of the amount and direction indicated. The distances to the other features follow from the basic rotation curve alone. The distance ambiguity has not been resolved for feature $\mathrm{H}$. The distances to the $\mathrm{HII}$ regions indicated are based on recombination line velocities (Mezger and Höglund, 1967). The absorption spectrum of the Hil region W51 (Burton, 1966b) shows dips at velocities corresponding to the distances indicated by the crosses. 
Irregularities in the terminal velocities are evident due to streaming in the sense of the density-wave theory and associated with the Sagittarius and Scutum arms. Two arms outside of the Sagittarius arm are included in the model; these two arms are too far from the galactic center to be seen tangentially so that only one branch appears on the model. One arm inside the Scutum arm is included in the model.

The parameters which are adjusted in constructing the model are the tilt angle of the two-arm exponential spiral and the density contrast of the gas, which is expressed as the ratio of the surface density at the axis of an arm to surface density in the interarm region.

The most important observations to be respected when adjusting the model parameters are the locations and amplitudes of the irregularities in the terminal velocities.

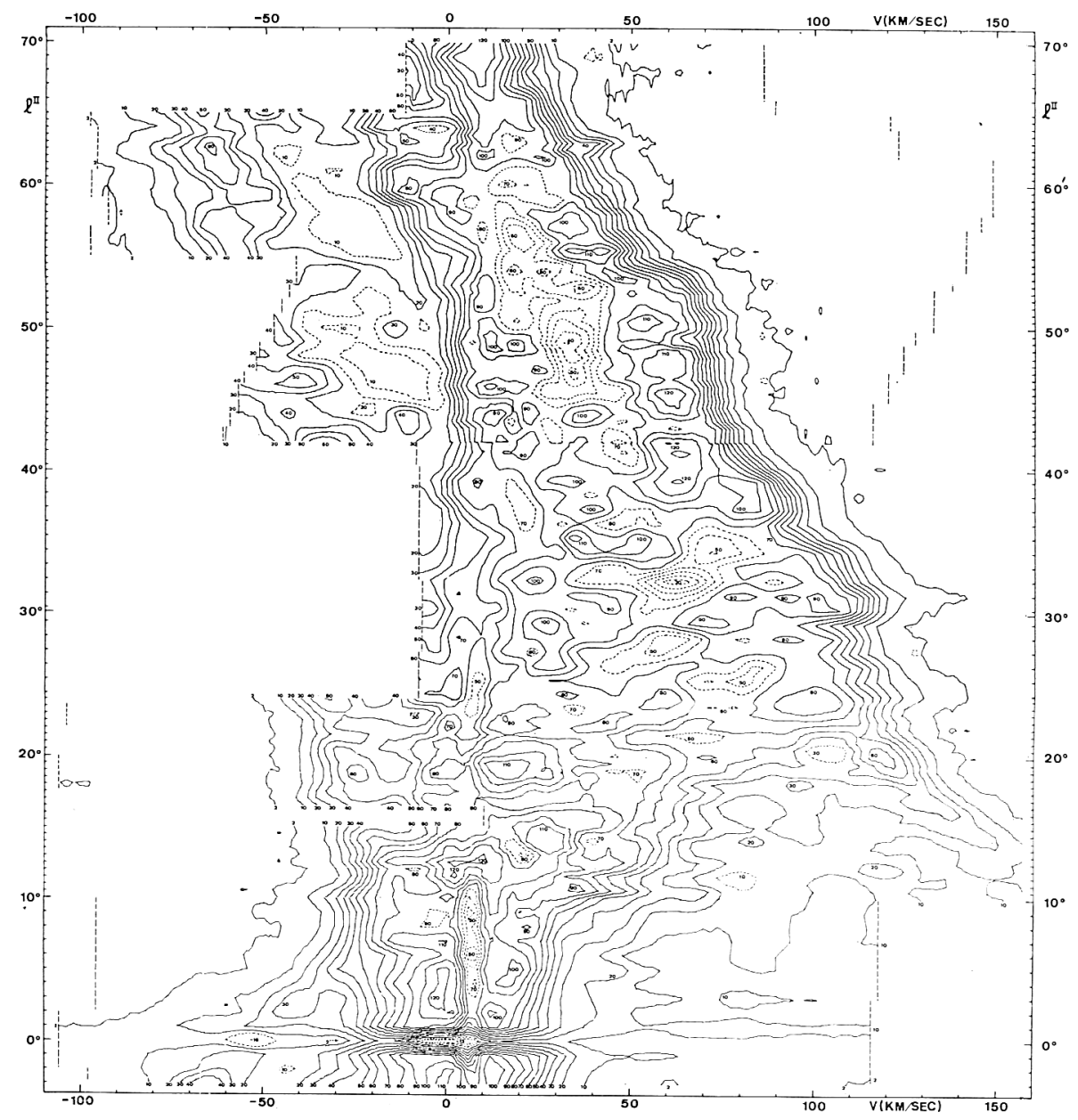

Fig. 4. Contour map of $\mathrm{HI}_{\mathrm{I}}$ brightness temperature in the galactic plane. The bandwidth is $2 \mathrm{~km} \mathrm{~s}^{-1}$ above $l^{11}=24^{\circ}$ and $4 \mathrm{~km} \mathrm{~s}^{-1}$ below. Contours were drawn at $2 \mathrm{~K}, 10 \mathrm{~K}, 20 \mathrm{~K}$, etc. Broken-line contours enclose regions of low brightness temperature. 
A scale factor puts the bump associated with the Sagittarius arm at the correct longitude of $50^{\circ}$. The tilt angle determines the longitude of the bump associated with the Scutum arm. If a constant value for the tilt is chosen, then the shape of the bumps is wrong and the body of the map agrees poorly with the observations, primarily because the other arms are not located in the correct positions. A better fit is obtained by taking the tilt angle to be a linear function of distance from the galactic center, such that the outer arms are more circular than the inner arms.

Once the tilt angle is specified, the amplitude of the bumps in the terminal velocities is determined by the gas density contrast parameter. The gas density contrast also

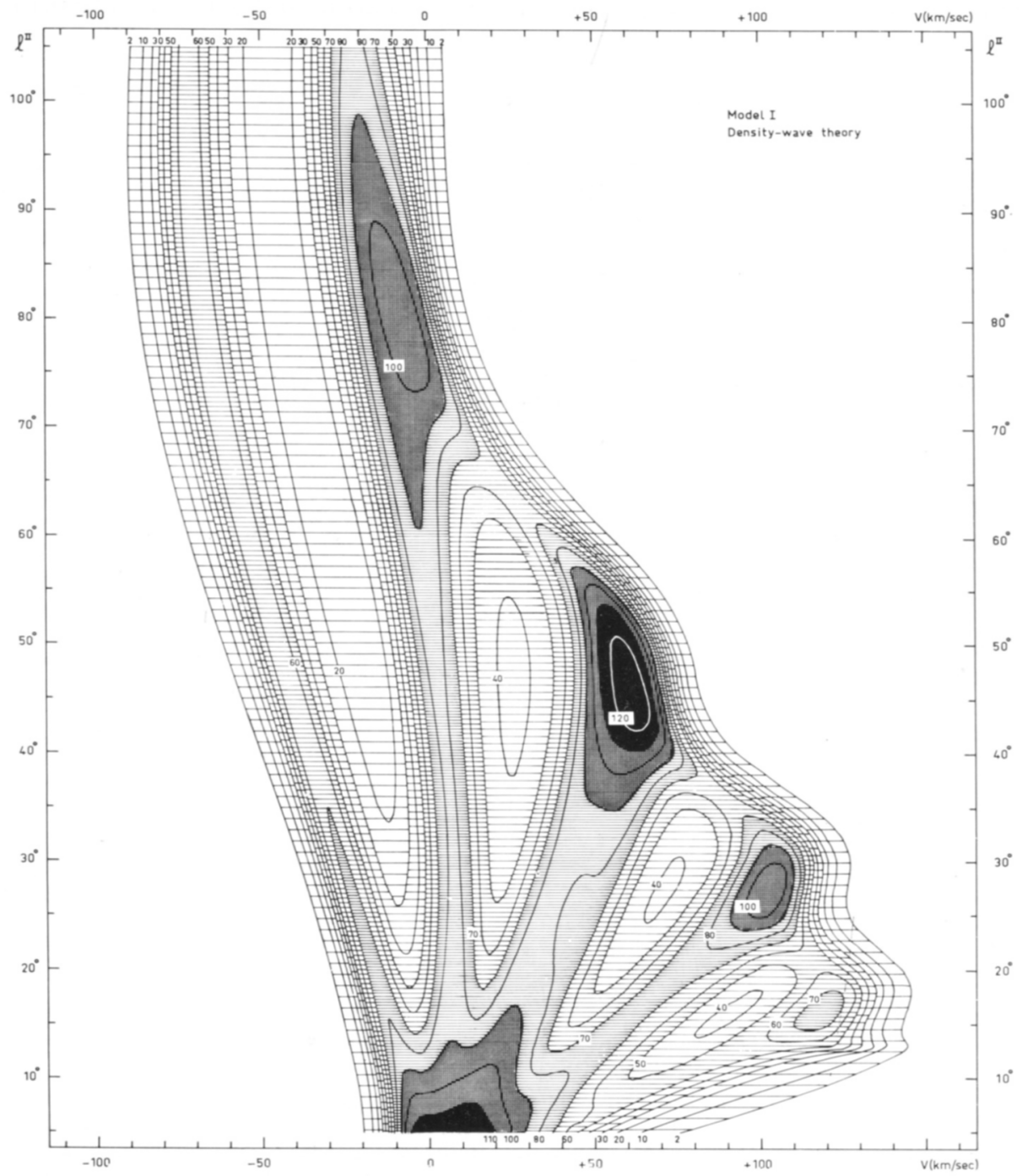

Fig. 5. Model I contour map for the galactic plane based on first-order density-wave theory. 
determines the relative intensities of peaks and valleys deep in the contour map. In the first-order density-wave theory the gas arms coincide with the stellar arms, and the gas density varies in a smoothly oscillating manner along galactic radii.

The tilt used in model I varies linearly from $8^{\circ}$ (with respect to a circle) at $5 \mathrm{kpc}$ from the galactic center to $5^{\circ}$ at $10 \mathrm{kpc}$. The contrast between gas density on the axis of an arm and interarm density varies linearly from 3:2 at $5 \mathrm{kpc}$ from the center to $3: 1$ at $10 \mathrm{kpc}$. The maximum streaming velocity predicted by the density-wave theory using this tilt and density contrast is about $8 \mathrm{~km} \mathrm{~s}^{-1}$. The density-wave pattern speed is held constant at $13 \mathrm{~km} \mathrm{~s}^{-1} \mathrm{kpc}^{-1}$. Formally the tilt angle is directly related to the pattern speed through the dispersion relationship of the density-wave theory. However, in constructing the model it was convenient to retain the tilt angle as a free parameter in finding a simple model with density-wave streaming in reasonable agreement with observations. Comparison of the pattern derived here with that

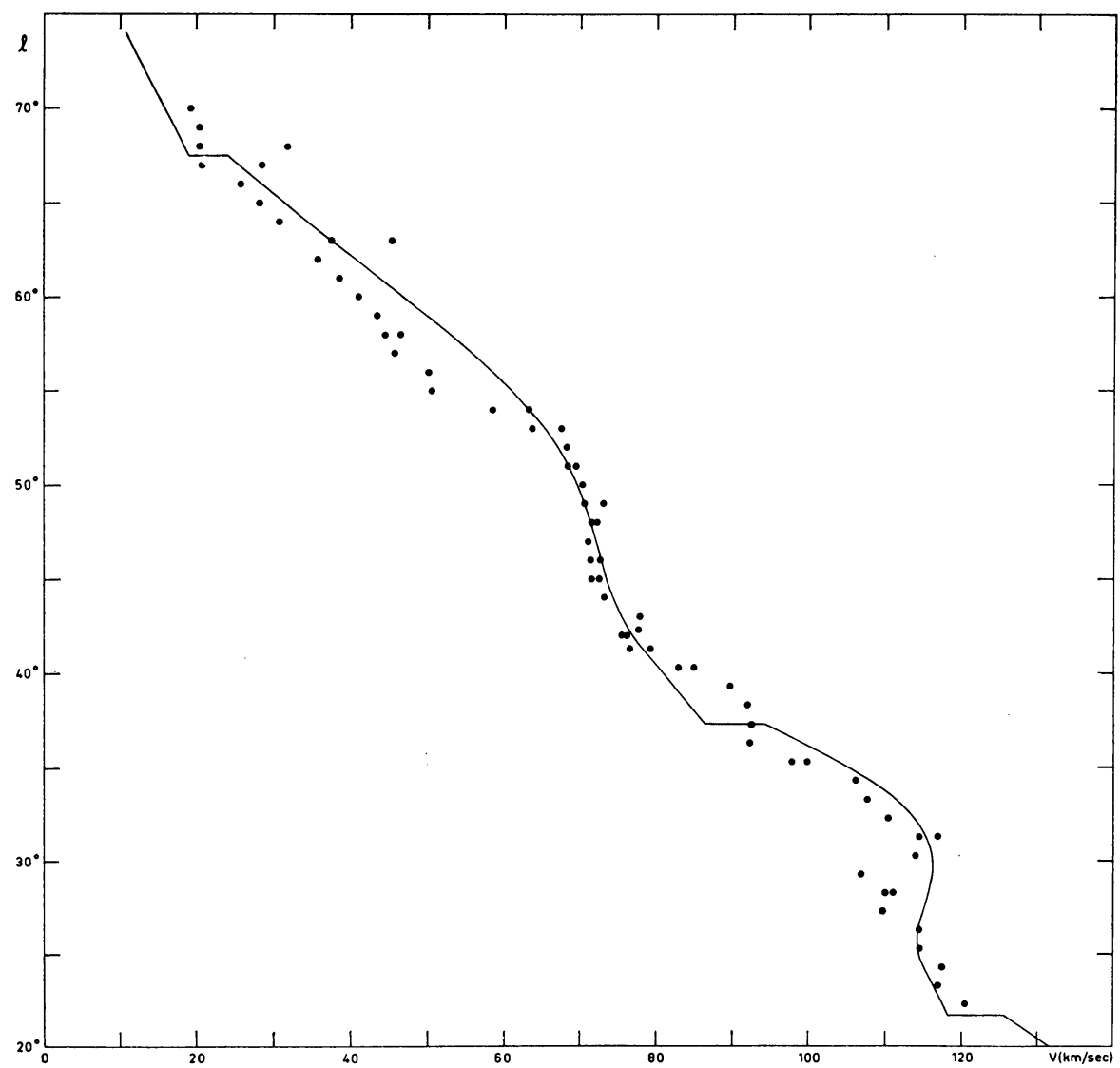

Fig. 6. Comparison of terminal velocities derived from observations and from model I. The dots are the observed terminal velocities measured by Shane and Bieger-Smith (1966). The line shows the terminal velocities calculated from model I. 
formally derived from the constant pattern speed of $13 \mathrm{~km} \mathrm{~s}^{-1} \mathrm{kpc}^{-1}$ showed that they agreed well, especially in the region under discussion.

The line profiles employed in the model contour map are smoothed with a velocity dispersion $\sigma_{v}=9.0-0.4 R\left(\mathrm{~km} \mathrm{~s}^{-1}\right)$. The average gas density is constant at $\left\langle n_{\mathrm{H}}\right\rangle=$ 0.4 atoms $\mathrm{cm}^{-3}$ for $R<10 \mathrm{kpc}$ and decreases linearly toward a cut-off at $R=25 \mathrm{kpc}$. A constant spin temperature $T_{s}=135 \mathrm{~K}$ was assumed.

The run of observed terminal velocities calculated by Shane and Bieger-Smith (1966) is compared in Figure 6 with the terminal velocities calculated in the same way from the profiles used in the model I contour map. The agreement is fairly good. The poorest agreement is in the region of the secondary bump at $l^{\mathrm{II}}=38^{\circ}$, which does not appear in the model, and near $l^{\mathrm{II}}=55^{\circ}$ where the observed velocities drop by more than $10 \mathrm{~km} \mathrm{~s}^{-1}$ in one degree of longitude. Abrupt changes in velocity or in density can not be explained by the first order density-wave theory. Sudden drops in terminal velocity derived from the model are due to the fact that at longitudes just below the drop the highest-velocity maxima are contributed by gas on the outside of one arm whereas at longitudes just above the drop they are contributed by gas streaming, in the opposite sense, along the inside of the next arm.

A comparison of the observed rotation curve with the unperturbed basic rotation curve and with that derived from model $I$ is given in Figure 7.

The geometry through which the space density distribution is transformed into the velocity-longitude relationships which we observe is complicated. The general ridge of intensities at high velocites in model I illustrates the effect of an extended path-

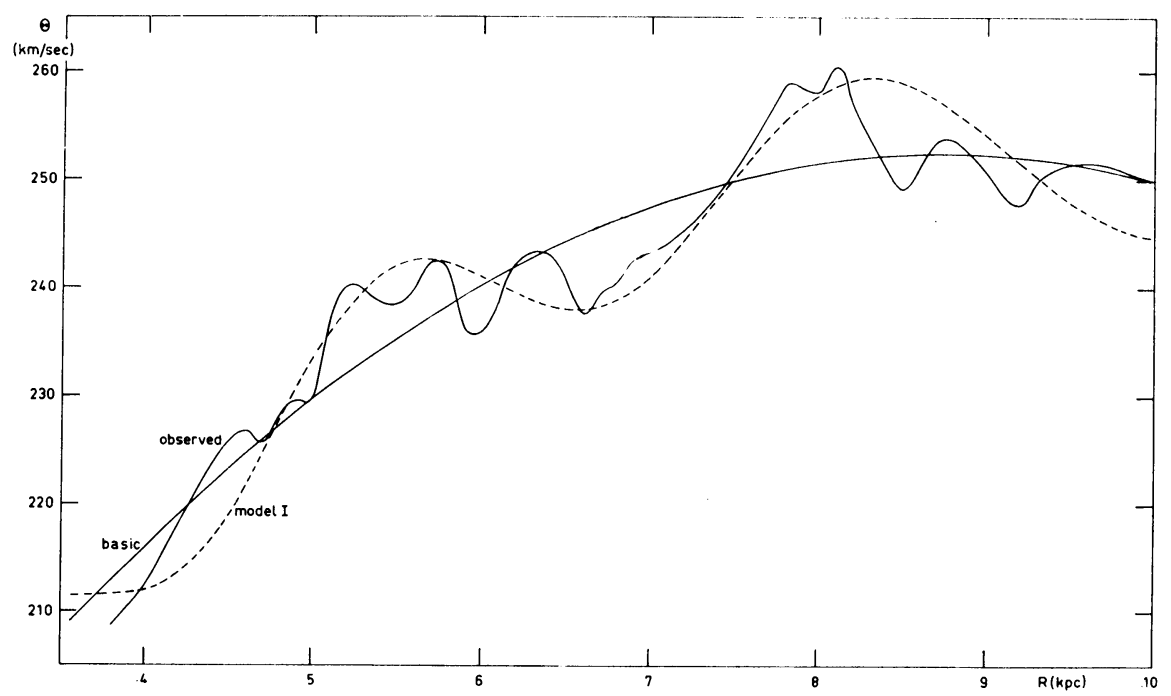

Fig. 7. Observed and computed apparent rotation curves. The irregular curve is that derived by Shane and Bieger-Smith (1966). The smooth curve is the basic rotation curve; the dashed one shows the basic rotation curve perturbed by the density-wave theory streamings of model I. In the presence of deviations from circular motion the maximum velocity might not come from the subcentral point; nevertheless the figure is drawn assuming that it does. 
length over which the velocity changes only slowly. Another example of the same situation is the very strong pattern in the model between $l^{\mathrm{II}}=65^{\circ}$ and $l^{\mathrm{II}}=90^{\circ}$, near zero velocity. The volume-density distribution is smooth along all the arms in the model; the changes as one moves along the axis of an arm in the velocity-longitude space of Figure 5 are due to crowding in velocity. One of the most important largescale characteristics of the observations shown in the reference map in Figure 4 is the general ridge of high velocity hydrogen. Furthermore, the observations show a strong pattern beginning at $l^{\mathrm{II}}=65^{\circ}$ and continuing to higher longitudes. These observed high intensities are due to real hydrogen, but not necessarily to a spatial concentration of hydrogen. This path-length effect must be kept in mind when converting the observed velocity-longitude patterns into a map of the Galaxy, in order to avoid spurious features on the map. The pattern labelled $\mathrm{H}$ in Figures 1 and 3 is a case in point. Above $l \approx 53^{\circ}$ this pattern appears as the high-velocity ridge on the observed contour maps. It is possible that these intensities are contributed by interarm gas which is not concentrated into a spiral-like fragment.

In reality, there are bound to be irregularities in the radial velocities as well as in the spatial structure. Large-scale irregularities of only a few $\mathrm{km} \mathrm{s}^{-1}$ in the radial velocities will have consequences in the profiles comparable to those due to irregularities in the structure.

In order to demonstrate the effects of streaming, model II has been calculated using the same density distribution as used in model $\mathrm{I}$, but using the basic rotation curve alone without the density-wave theory perturbations. The contour map of this circular rotation model is shown in Figure 8 . Model II has a strikingly different appearance from model I, even though the input space distribution is exactly the same in both models. Of course the run of terminal velocities in model II is quite smooth. The slope of the major patterns in the model contour maps is partly determined by the amount of streaming. The slopes of the patterns in the density-wave theory model agree better with the observations than the slopes in the circular rotation model.

Model III, shown in Figure 9, is based on the assumptions of circular rotation and no gas between arms. The distribution of the average density $\left\langle n_{\mathrm{H}}\right\rangle$ is the same in this model as in the previous ones. The hypothesis of no gas between arms is unlikely. Furthermore the model contour map shows that these assumptions also cannot represent the observations, the major failures being that the shape of the irregularities in the run of terminal velocities is all wrong, and that the high-velocity ridge of hydrogen, so characteristic of the observations, is missing in model III.

The sort of insights which can be gained from model calculations into the complicated relationships between space distribution and velocity-longitude diagrams leads us to suggest that it might prove useful to calculate model line profiles whenever one produces a map of the spiral structure. These profiles, which would be based on the velocity field used to derive the kinematic distances in the map, would then be compared with the observations to test if the spiral structure map is a reasonable one.

It is clear that attempts to derive the velocity field must be based on simultaneously 
derived solutions for the distribution of the gas. In the first step outlined above, the emphasis was on the hydrogen distribution. In the second and third steps the emphasis was on the velocity field. The density-wave theory provides a velocity field in better agreement with the observations than that provided by circular rotation.

With a better velocity field, can we draw a better map of the Galaxy? The velocity field derived from fitting the density-wave theory to the observations already implies a mass distribution. Since the theoretical model clearly does not represent the observations perfectly, and in some regions it fails grossly, a map based on the velocity-field

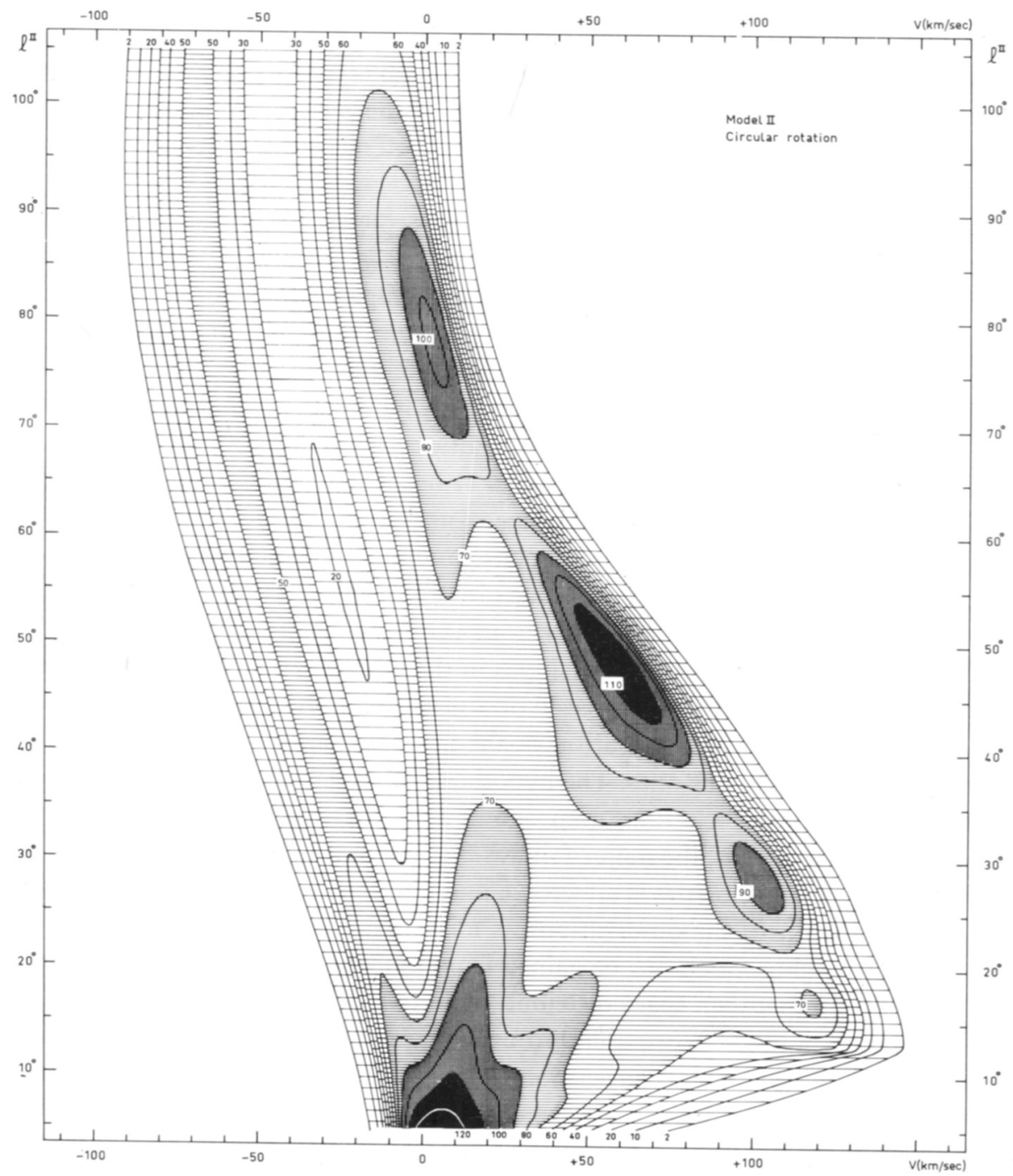

Fig. 8. Model II contour map based on circular rotation. The same density distribution is used in this model as in model $\mathrm{I}$. 
of the model will place some features in space where they contradict the space distribution used as input in deriving the velocity field.

It seems best as a next step to take the insights we have won into the velocity field and to go back to the observations, but now with the emphasis again on the hydrogen density distribution.

\section{The Velocity Field in the Scutum Arm}

In order to study more closely the properties of the models it is useful to compare the velocity-longitude diagram observed with that corresponding to the density

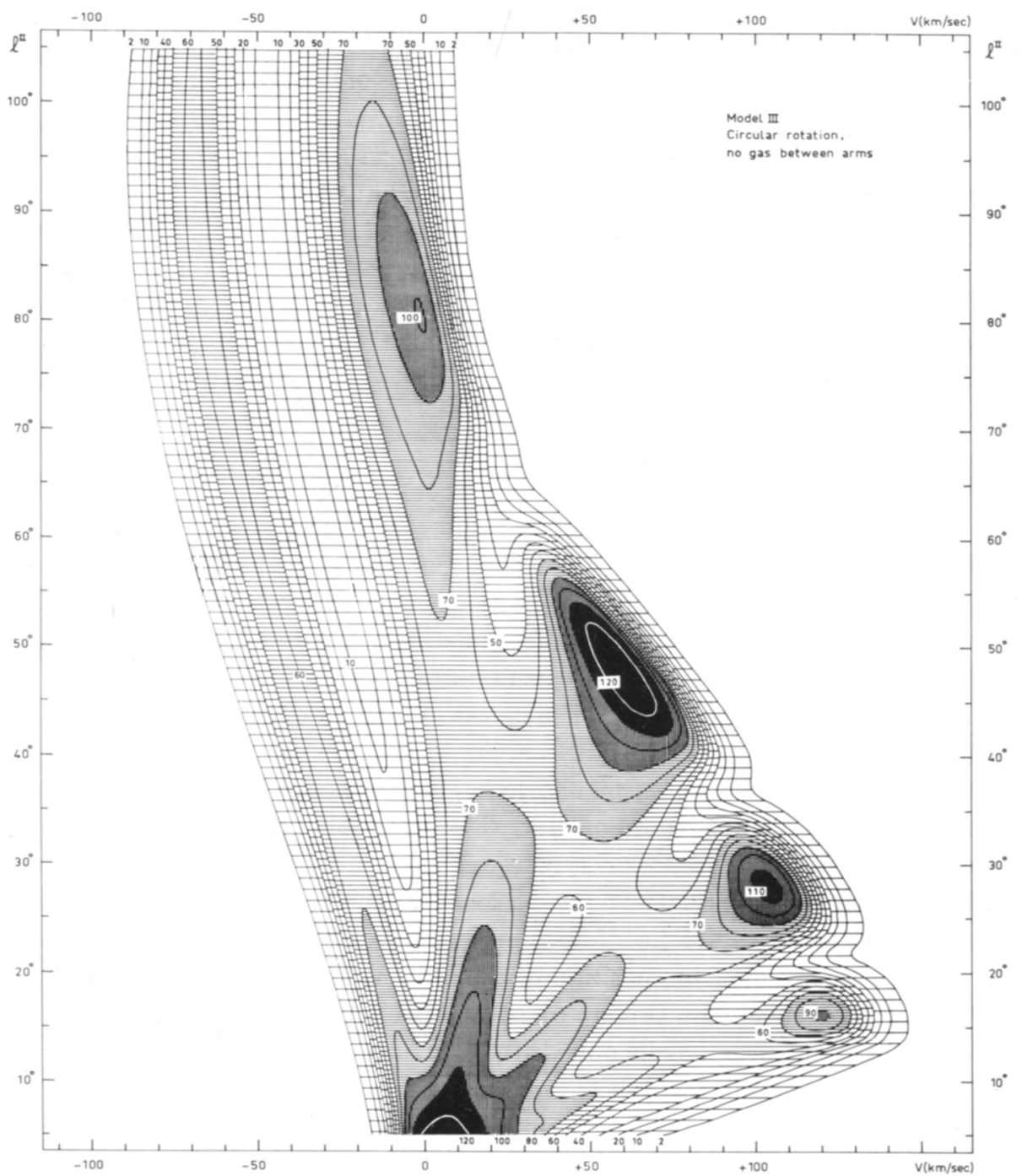

Fig. 9. Model III contour map based on circular rotation and no gas between arms. 
maxima of the model. This method has the advantage that the most important features of the whole observational material are presented in a simple way. The comparison must be made with caution, however, as dissimilar features are being plotted; for example, the persistent intensity maximum at high velocity which is such a prominent feature of all the contour maps is absent from the model results as shown in this way.

In Figure 1 we have plotted the axes of the Sagittarius and Scutum arms computed for model I as broken curves. The agreement with the observations is not very satisfactory. Considering the Scutum arm, we see that the loop of the arm derived from the model is far too closed and is located at too low a longitude. In order to open the loop we may try to increase the tilt of the arm. This is an inefficient process, since the density-wave streaming velocities will increase with the tilt and will work to close the loop, so that a tilt of more then $20^{\circ}$ would be required to produce the observed figure. Such high tilts are not expected in the neighbourhood of the inner Lindblad resonance, and it would be necessary to introduce a very rapid increase in tilt inward from the Sagittarius arm and probably a corresponding increase in the multiplicity of arms.

An alternative possibility is to introduce a radial expansion in the region of the Scutum arm. Such an assumption introduces dynamical difficulties and it is questionable if the first-order density-wave theory could have any validity in the presence of such an expansion. The hypothesis, however, serves to represent these observations and is supported by three independent observational results; these are the expansion of the 3-kpc arm as seen in absorption, the rapid increase in the observed maximum velocity which sets in for longitudes lower than about $l^{\mathrm{II}}=22^{\circ}$ and which could be explained otherwise only by a very sharp break in the rotation curve, and the extension of the far (i.e. higher velocity) branch of the Scutum arm to $l^{\mathrm{II}}=0^{\circ}$ at the velocity of $+70 \mathrm{~km} \mathrm{~s}^{-1}$.

For the reasons given above we favor the expansion model, which we call number IV, over one with rapidly changing tilt, and have made the corresponding calculation. We have used the same parameters as in model I but have introduced an additional axisymmetric expansion, given, as a function of distance from the galactic center, in the table in Figure 10. The behavior assigned at distances less than $2 \mathrm{kpc}$ from the center is quite arbitrary. The velocity-longitude diagram of the axis of the Scutum arm as calculated from this model is shown in Figure 1 as a dotted curve. The Sagittarius arm, lying outside the region of expansion, is unchanged from model I.

We see that this model represents quite well the opening of the Scutum arm loop and the extension to $l^{\mathrm{II}}=0^{\circ}$. The assumed location of the Scutum arm in the model, however, is unaltered, and it lies several hundred parsecs inward from the observed location. We cannot alter the position of the arm in the model without spoiling the fit to the rotation curve data (Figure 6), so we conclude that the main concentration of gas in the Scutum arm may lie outside the axis of the mass concentration. A secondary arc, lying inside the axis of the arm, is also seen in Figure 1. This loop is even more open than that of the main arm, implying a higher expansion velocity, in agreement with model IV. The contour map corresponding to model IV is shown in Figure 10. Comparison of this map with Figure 4 reveals not only the similarity in behavior 


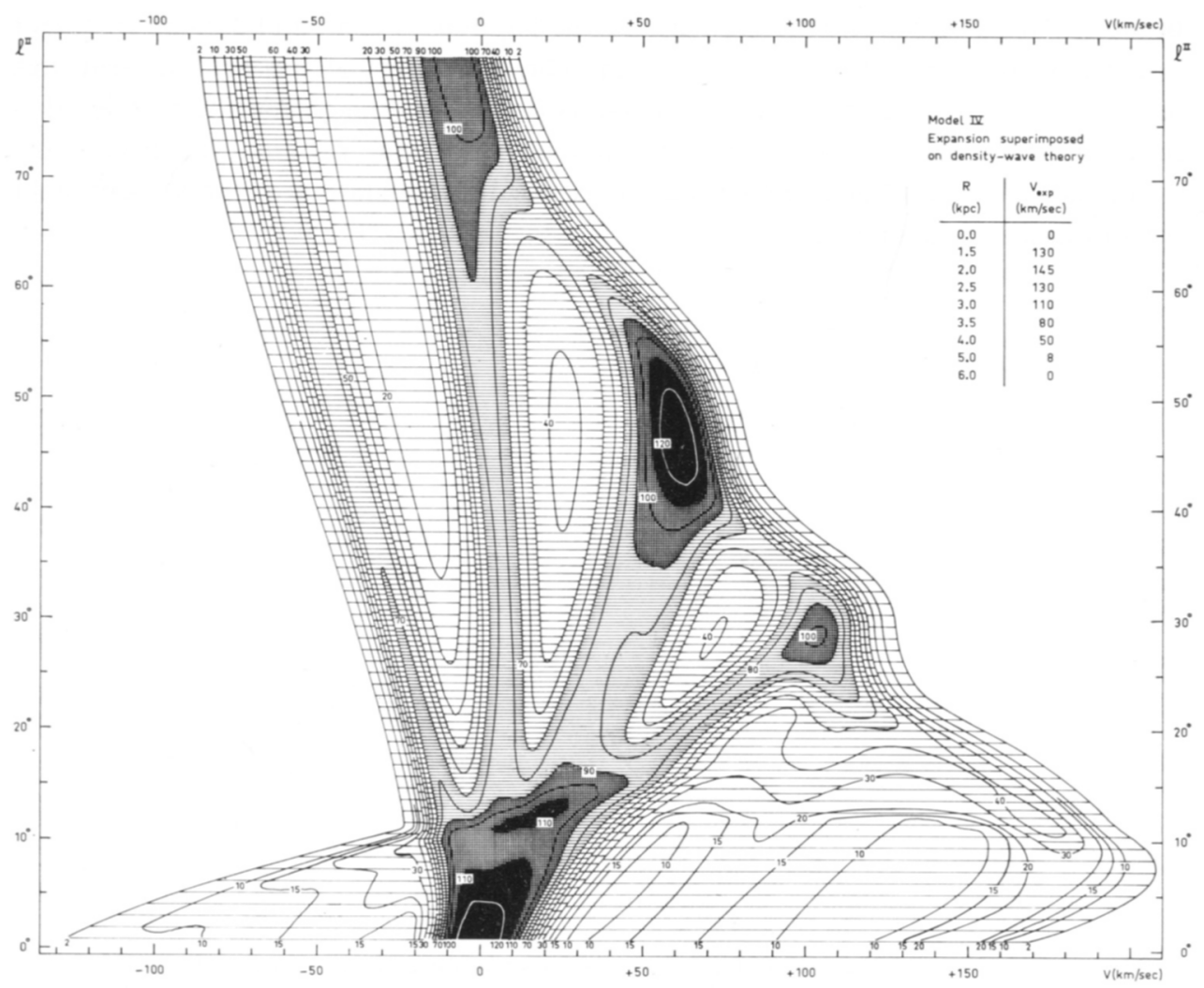

Fig. 10. Model IV contour map based on the same assumptions as model I but with the radial expansion, given in the table, superimposed on the other motions.

of the Scutum arm but also general agreement of the maximum velocity at $l^{\mathrm{II}}<22^{\circ}$ and a small feature at negative velocity close to $l^{\mathrm{II}}=0^{\circ}$ which may correspond to the 3-kpc arm.

In order to examine further the doubling of the Scutum arm, we have compared the maximum brightness temperatures at high velocity as observed and as computed from model IV. These are plotted in Figure 11 for the longitude range $22^{\circ} \leqslant l^{\mathrm{II}} \leqslant 42^{\circ}$. The rise toward the maximum of the Sagittarius arm is accurately expressed by the model, but the broad maximum of the Scutum arm shown in the model is clearly split in the observations. In this representation the inner and outer parts appear to be of about equal strength.

\section{The Distribution of the Neutral Hydrogen}

Using the velocity field of model IV we have constructed a map (Figure 12) of the inner region of the Galaxy in the range $l^{\mathrm{II}}=22^{\circ} .3$ to $42^{\circ} .3$. We see the arcs belonging to the Scutum arm as well as a small feature which may be associated with the far 
branch of the outer arc, both branches of the Sagittarius arm, and a small fragment which may belong to the next outer arm. The extensions of the Scutum arm arcs outward along the locus of subcentral points are due to the high-velocity ridge discussed above and do not necessarily represent a spatial concentration of $\mathrm{HI}$. The axes of the Sagittarius and Scutum arms assumed for the calculation of the velocity field are shown as broken curves.

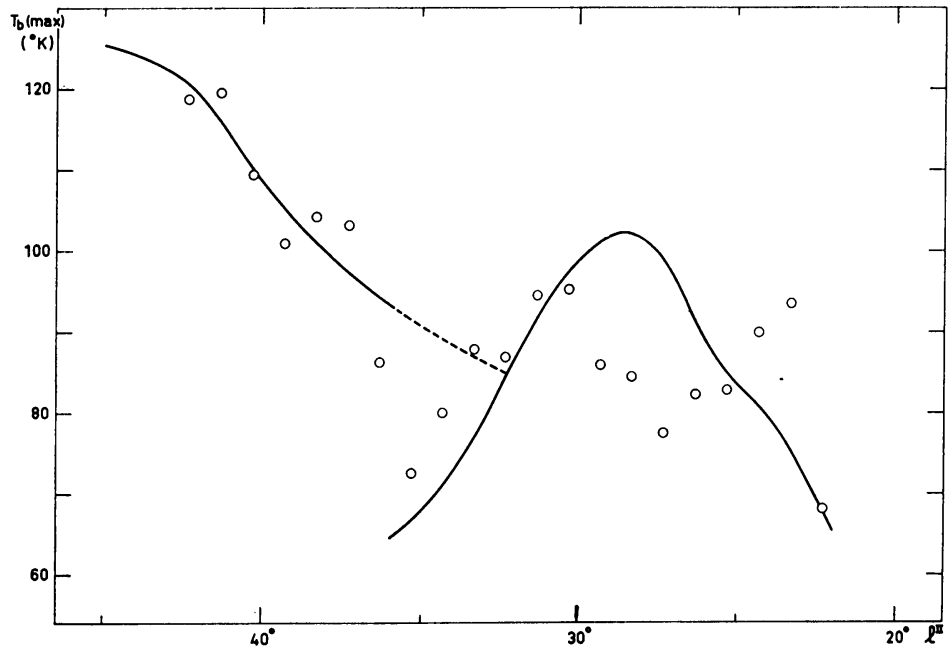

Fig. 11. Comparison of the maximum brightness temperature at high velocity observed (circles) and calculated from model IV (curve); the broken part of the curve represents an alternative choice for the velocity of the maximum.

It is tempting to compare the location of the $\mathrm{HI}$ as plotted in Figure 12 with that of the arms as introduced, mainly from dynamical considerations, into the model calculations. For two reasons one must be very cautious about drawing conclusions from such a comparison. In the first place, the position of the arms assumed for the model calculations is determined largely from the evidence of the rotation curve which pertains only to the neighbourhood of the subcentral points, and from which the location throughout the region is inferred. A perturbation near this locus may result in a substantial shift of the assumed position of the whole arm. In the second place, the first-order density-wave theory does not pretend to give an accurate picture of either the density or the velocity field within an arm. Recent calculations by Roberts (1969) have, in fact, shown that substantial deviations from the first-order theory may be expected. Thus kinematic distances derived from the first-order theory may contain systematic errors of the order of the dimensions of an arm. Furthermore the first-order theory can hardly be expected to be valid under the conditions of expansion assumed here. The presence of the deformation in the rotation curve in the neighbourhood of the Scutum arm indicates, however, that the velocity 
field is in fact perturbed in a manner similar to that predicted by the first-order theory, which then serves as a means of extrapolation.

Bearing in mind the above limitations, we can nevertheless draw some conclusions about the distribution of $\mathrm{HI}_{\mathrm{I}}$ in the region considered. It appears that the Scutum arm is divided into two arcs separated by almost $1 \mathrm{kpc}$. The gas in both arcs appears to be moving outward from the galactic center with velocities approximately equal to those given in the table in Figure 10. The appearance of the Scutum arm in the velocity-

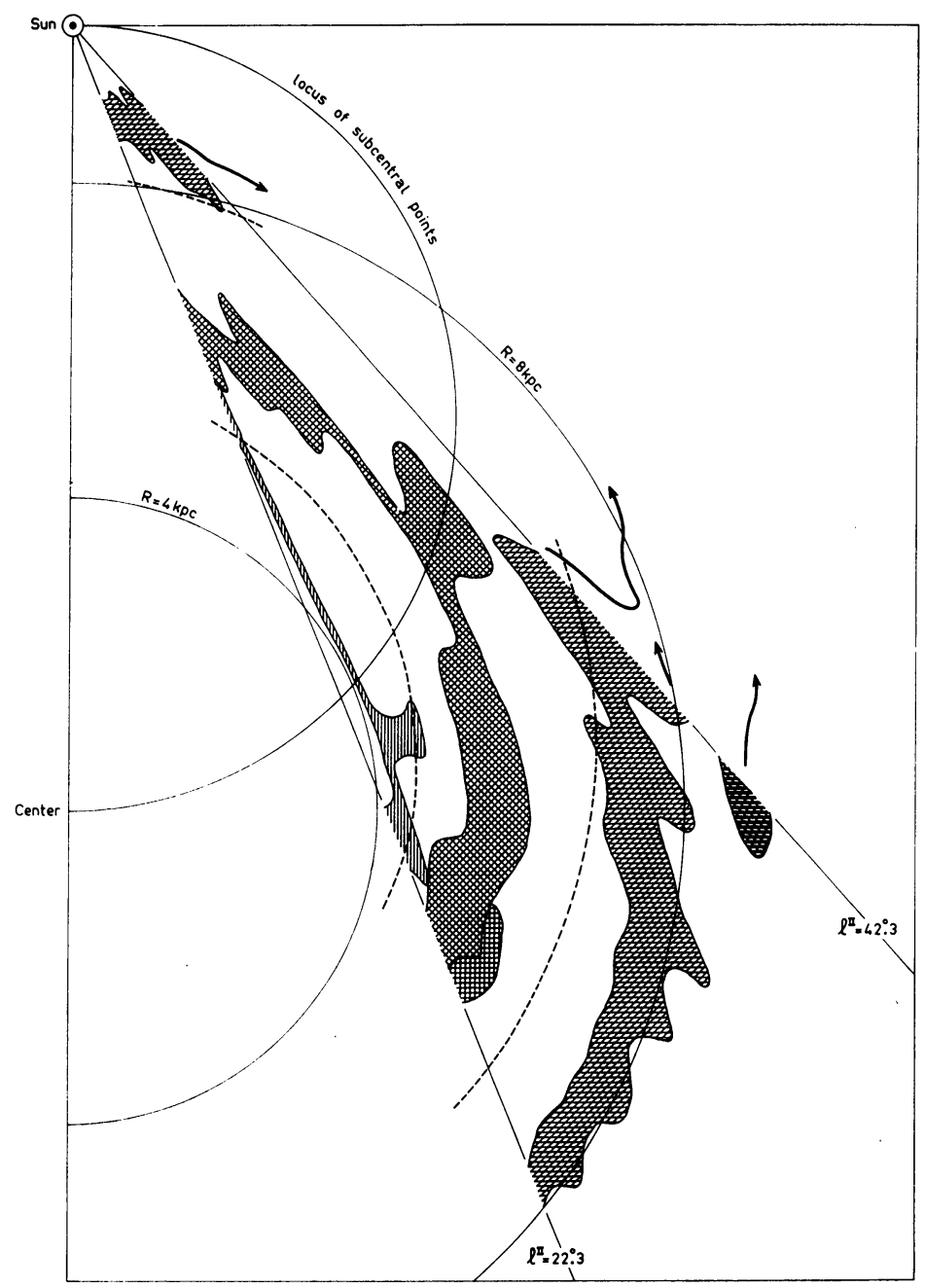

Fig. 12. Map of the $\mathrm{H}_{\mathrm{I}}$ distribution near the galactic plane in the region $22^{\circ} .3 \leqslant l^{11} \leqslant 42^{\circ} .3$. The kinematic distances are based on model IV. The breadth of each feature represents the HI content (not its spatial extent), $\sigma_{v} \tau=5 \mathrm{~km} \mathrm{~s}^{-1}$ being represented by $1 \mathrm{kpc}$ along the line of sight. The shading is the same as that used in Figure 1. The extension into the higher longitude region is indicated schematically. The broken curves indicate the locations of the axes of the Sagittarius and Scutum arms adopted in all of the model calculations. 
longitude diagram supports this conclusion. The Sagittarius arm as plotted does not closely resemble a spiral, the far branch being almost circular and the near branch quite sharply inclined. This suggests a large scale asymmetry in either the velocity field or the density distribution or both, but in view of the limitations discussed in the preceding paragraph such a conclusion cannot be drawn with any confidence on the basis of the present model calculations.

\section{References}

Burton, W. B.: 1966a, Bull. Astron. Inst. Netherl. 18, 247.

Burton, W. B.: 1966b, Astron. J. 71, 848.

Kwee, K. K., Muller. C. A., and Westerhout, G.: 1954, Bull. Astron. Inst. Netherl. 12, 211.

Lin, C. C., Shu, F. H., and Yuan, C.: 1969, Astrophys. J. 155, 721.

Mezger, P. G. and Höglund, B.: 1967, Astrophys. J. 147, 490.

Roberts, W. W.: 1969, Astrophys. J. 158, 123.

Shane, W. W. and Bieger-Smith, G. P.: 1966, Bull. Astron. Inst. Netherl. 18, 263. 